\title{
Features of destruction of a ferrite-martensitic composite
}

\author{
Viktor N. Pustovoit, Valentina V. Duka, Yuri V. Dolgachev", Ludmila P. Aref'eva, \\ Vladislav $V$. Fedosov and Viktoria M. Salynskih \\ Don State Technical University, Physical and Applied Materials Science Department, 344000 \\ Rostov-on-Don, Russia
}

\begin{abstract}
The behavior of a crack in steel with the structure of a ferriticmartensitic composite is studied. The modes of heat treatment for obtaining a composite structure with alternating layers of ferrite and martensite are given. The kinetics of crack development during cyclic tests was studied. The obtained data show that the rate of development of the crack in the material with the structure of the composite is substantially lower than for the usual sorbite structure. It is shown that the frontal growth of the crack in the composite is carried out with "stops", on which the matrix deforms and its stratification occurs in the direction normal to the front of the crack motion.
\end{abstract}

\section{Introduction}

The steel used in construction must reliably work in a complex combination of force and temperature fields and the influence of an aggressive environment. Along with this, they must have a high yield strength, high structural strength and manufacturability. The use of materials combining high plastic deformation resistance and high values of fracture resistance characteristics is relevant for high-loaded construction and bridge structures, highpressure boilers and tubular products. The propensity of materials to destruction is caused by the presence of metallurgical defects (microcracks, nonmetallic inclusions) and the formation of fracture-like defects during operation.

The purpose of this work is to study the behavior of a crack in samples of A414(F) steel with the structure of a natural ferritic-martensitic composite (NFMC). For comparison, samples were selected from the same steel with the structure of tempering sorbite.

The previous technology of obtaining the structure of the NFMC [1-3] assumed the plastic deformation of hypoeutectoid steel in the intercritical temperature interval $A_{c l} \div A_{c 3}$ to obtain an oriented austenite-ferrite structure and subsequent quenching. As a result, an oriented ferritic-martensitic structure is formed. The shortcomings of this technology are obvious: powerful press equipment is needed (low heating temperature), it is impossible to obtain a composite in the finished product, it is problematic to obtain a sufficiently strict orientation of martensite and ferrite along the axis of deformation. At the same time, the misorientation

\footnotetext{
* Corresponding author: yuridol@mail.ru
} 
should not be more than $10 \div 15^{\circ}$, as this largely determines the mechanism for transferring the stresses from the soft matrix to the strong fiber and the type of failure of the composite.

These disadvantages are eliminated by using the processing technology described in the patent [4], which assumes the use of steels with an already existing fibrous structure to create an NFMC. The proposed method for creating a natural ferritic-martensitic composite eliminates the need for deformation processes. It is based on the use of hot-rolled hypoeutectoid steels with banded ferritic-perlite structure (Fig. 1), which results from delayed cooling of rolled products and formation of ferrite along the rolling direction. In this case, a structure is formed from alternating ferrite and perlite bands, the thickness of which depends on the cooling rate of the rolled product. After heating such a steel into the intercritical region and quenching, the structure of the NFMC appears.

\section{Methods of study}

In the experiments we used samples from A414(F) steel. Their chemical composition was refined with the help of the Q8 Magellan optical emission spectrometer. The average quantities of chemical elements are given in Table 1.

Table 1. The chemical composition of samples from steel A414 (F).

\begin{tabular}{|c|c|c|c|c|c|c|c|c|c|}
\hline \multicolumn{10}{|c|}{ Content of elements, \% by weight } \\
\hline $\mathbf{C}$ & $\mathbf{S i}$ & $\mathbf{M n}$ & $\mathbf{S}$ & $\mathbf{P}$ & $\mathbf{C r}$ & $\mathbf{M o}$ & $\mathbf{N i}$ & Al & Co \\
\hline 0.142 & 0.366 & 1.432 & 0.017 & 0.023 & 0.270 & 0.005 & 0.115 & 0.002 & 0.021 \\
\hline
\end{tabular}

A ferritic-martensitic composite was obtained by heating in the intercritical temperature range $A_{c l} \div A_{c 3}$ in which an exposure was carried out, which is necessary for refining ferrite and producing an austenite with a significantly higher carbon content than in the original steel. To obtain the necessary ratio of martensite and ferrite, a certain quenching temperature was selected. Selection of the quenching temperature was carried out approximately in the interval $730-870{ }^{\circ} \mathrm{C}$ according to the state diagram, and then experimentally by metallographic studies.

As a result, a temperature of $730^{\circ} \mathrm{C}$ was chosen which forms a structure containing $25-30 \%$ martensite (Fig. 2). With a larger volume fraction of martensite, the work of destruction decreases [5], since an increase in the yield strength and a decrease in the size of the plastic deformation zone of the matrix occurs.

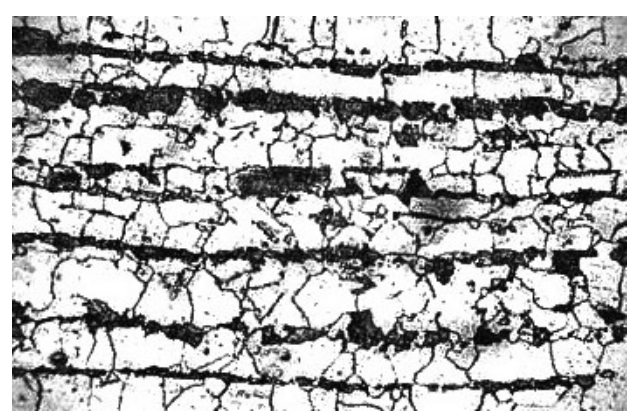

Fig. 1. Striped ferritic-pearlite structure of A414 (F) steel, $\times 200$.

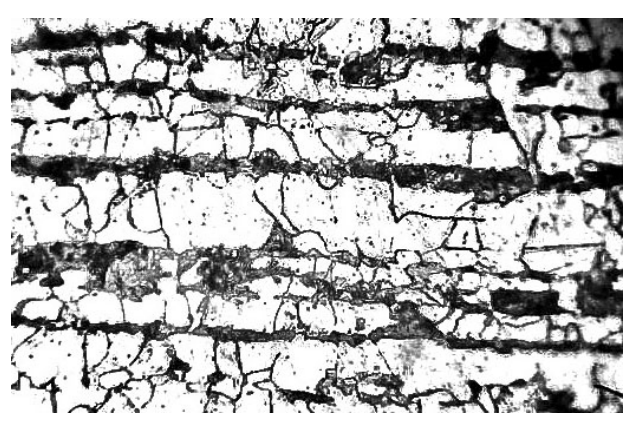

Fig. 2. Steel with the structure of NFMC ( $29 \%$ martensite),$\times 360$.

Investigation of the kinetics of crack development was carried out during cyclic tests of prismatic specimens $(10 \times 11 \mathrm{~mm})$ with a notch $(r=0.25 \mathrm{~mm}$, depth $1 \mathrm{~mm})$ on a special vibro 
unit. The vibration unit works according to the scheme of rotation of the unbalanced mass, while the sample undergoes an asymmetric bending. Observation of the formation of a crack was carried out using a binocular microscope. Fixation of crack development was realized using the method of electro potentials $[6,7]$ in the coordinates "potential difference - time". Then the dependence was deciphered according to the calibration curve for plotting the curves "crack length - number of load cycles". The change in the rate of crack growth at different sections of its length was determined by graphically differentiating the obtained dependencies.

\section{Results and discussion}

The fig. 3 shows the results obtained. Steel with structure of NFMC destroyed after $90 \cdot 10^{4}$ test cycles, while tempering steel with sorbite structure withstands only $78 \cdot 10^{4}$ cycles. In both cases, the process of crack formation in the notch is structurally insensitive. The growth of the crack at the initial stage of formation occurs at the same rate, but further the kinetics of the development of the crack differs significantly for the two compared structures.

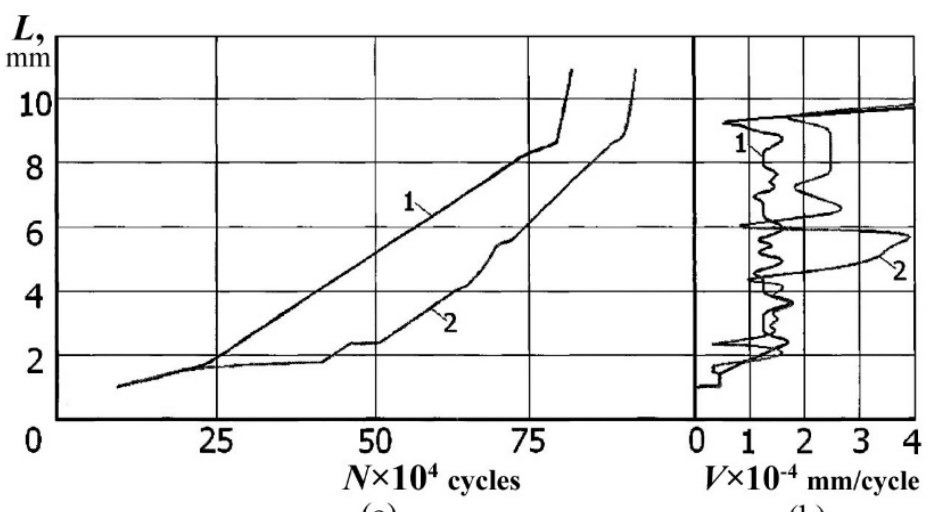

(a)

(b)

Fig. 3. Dependence of crack length on the number of cycles (a) and the crack growth rate at different sections of its trajectory (b): 1 - structure of tempering sorbite; 2 - structure of the NFMC.

The crack in a sample with a sorbite structure grows at a rate approximately the same (see Fig. 3, b) until destruction, when the "living cross section" is $\sim 2.5 \mathrm{~mm}$. For steel with an NFMC structure, the crack does not grow along the front in the range of $20 \cdot 10^{4}$ to $40 \cdot 10^{4}$ cycles. In the following, the crack grows along the front. However, in the process of increasing the length of the crack, stops are observed when the growth rate is almost zero. At the same time, in some sections of the crack propagation along the front, the rate of its growth is much higher than in steel with the structure of tempering sorbite. The destruction of the NFMC occurs at the remaining "live cross section" $\sim 2.3 \mathrm{~mm}$.

Such a behavior of the crack in a sample with the structure of the NFMC is due to a specific mechanism of failure in the composite having a soft matrix and strong fibers. The phenomenology of this mechanism is similar to the mechanism of destruction of composite materials [5] and steels with a structure of lamellar perlite [8].

In both cases for sorbite and composite, the primary crack is formed due to the destruction of carbides or martensitic plates closest to the sharp incision. Further in the composite the frontal growth of the crack stops, which is connected with the relaxation of stresses in its mouth. Stresses cause intense plastic deformation in ferrite bands. It can be assumed that the shift in the ferrite occurs not only in microvolumes adjacent to the mouth of the primary 
crack, but also in microvolumes sufficiently far from the mouth. The mechanism of viscous fracture during plastic shear of ferrite plates works by nucleation, growth and coalescence of pores. Relaxation of crack stresses in ferrite leads to the fact that frontal crack growth stops during a sufficiently large number of test cycles. The transition of a crack from one ferritic gap to another is due to the fact that strong fibers are discrete, i.e. there are gaps between adjacent ferrite fibers. In strong fibers, the stress does not exceed the elastic limit.

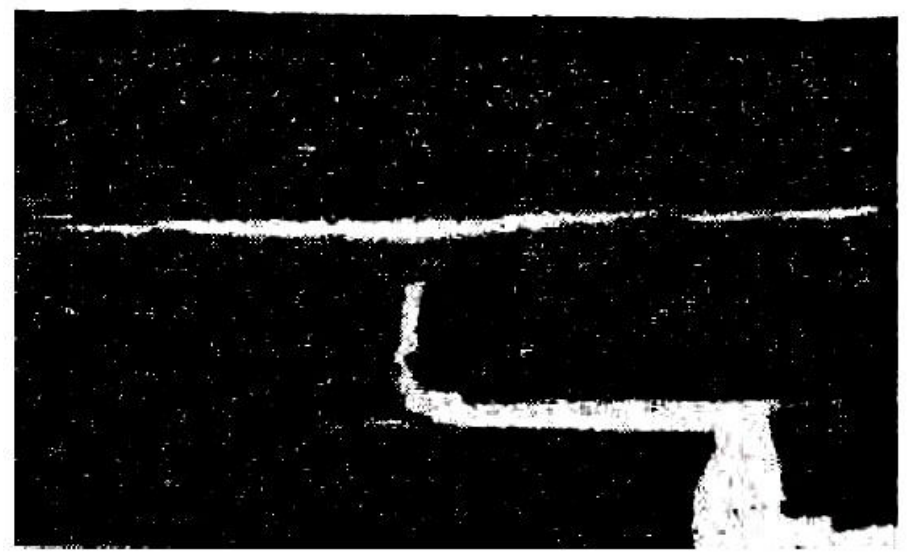

Fig. 4. Destruction at the end of the incision in the composite (stratification along the fiber) [5].

Let us consider the mechanism of pore nucleation in ferrite. When fatigue loading under conditions of alternating bending in ferrite, a significant number of vacancies are formed. They are formed at the intersection of dislocations, as well as when dislocations slide with thresholds [1]. The union of individual vacancies with formation of a pore occurs in the slip plane. Under the action of normal stresses on the surface of micropores, vacancies are deposited. Gradually the pore transforms into a crack [9]. In this case, there is a situation where there is almost complete insensitivity to the incision (with the initial crack formed). This is due to the development of the process of stratification in the direction of parallel fibers (Fig. 4).

The contribution of the work of deformation of the matrix to the overall work of fracture of the composite is proportional to the energy (per unit volume) spent on plastic deformation of the matrix prior to its destruction. In this case, the energy expended must be multiplied by the volume of the matrix, which was deformed when a unit of the crack surface was formed. Such a deformed volume is $V_{f} \cdot h$ [5], where $V_{f}$ is the volume fraction of the matrix (ferrite); $h$ is the distance between the cracks in the matrix:

$$
h=\frac{V_{f}}{V_{m}} \cdot \frac{r \cdot \sigma_{f}}{2 \cdot \tau}
$$

where $V_{m}$ is the volume fraction of strong fibers (martensite); $r$ is the radius of a strong fiber; $\sigma_{f}$ is the ultimate strength of the matrix; $\tau$ is the tangential stress at the interface between the strong fiber and the matrix, which causes the plastic flow of the matrix. The total work of destruction of the composite is proportional to $\frac{V_{f}^{2} \cdot r}{V_{m}}$. Obviously, it grows with increasing $r$, and the value of $V_{m}$ should be greater than $V_{\text {crit. }}$ [5].

The motion of the crack along the front occurs after a sufficiently long period of insensitivity to the incision (along with the primary crack), which amounted to at least $20 \cdot 10^{4}$ test cycles. This is due to the peculiarities of the mechanism of cracking of strong martensitic fibers. The mechanism of cracking involves the destruction of fibers as a result of the creation 
of high local stresses on the interface between ferrite and martensite. These stresses arise from the accumulation of dislocations coming from the ferrite (in which the deformation begins in the first place). Another feature of cracking is that strong fibers are separated from the matrix, which contributes to brittle fracture under the action of normal stresses. Normal stresses are created by external loading (in this case, $\tau$ decreases almost to zero). Passing through strong fibers, the crack moves along the front at a high speed (see Figure 3, b) until it encounters an undeformed volume of the matrix in its path. In this case, frontal growth stops, and then everything repeats, as described above.

\section{Conclusions}

In a material with an NFMC structure, crack growth in the front direction is effected by jumps, which alternate with delays. During the delays, the matrix deforms and its stratification occurs in the direction normal to the crack front. The mechanism of destruction in a material with an NFMC structure requires a large expenditure of energy than in a material whose structure does not provide anisotropy of fracture propagation resistance.

\section{References}

1. M.L. Bernshtejn, L.M. Kaputkina, V.G. Prokoshkina, V.A. Varganov, G.S. Krivonogov, A.S. Klyuch, Izv. Akad. Nauk SSSR, Metal., 13, 107 (1981)

2. I.N. Kidin, V.I. Lizunov, V.M. Belyavskaya, Met. Sci. Heat. Treat., 16, 308 (1974)

3. R.A. Grange, 2nd Int. Conf. of Strength of Metals and Alloys, 3, 861 (1970)

4. V.N. Pustovoit, Yu.M. Dombrovskii, A.V. Zheleva M.V. Zayitceva, RU Patent No. 2495141 (2012)

5. A. Kelly, Concise encyclopedia of composite materials (Elsevier, 2012)

6. S.A. Grishin Progressive Methods of Thermal Hardening in Tractor and Agricultural Machine Building, 15 (Rostov-on-Don, 1982)

7. V.L. Sorokin, V.F Zmitruk, G.V. Shcherbedinskii, V.S. Urusov, V.I. Sarrak, Zavod. Lab., 47(12), 60 (1981)

8. L.E. Miller, G.S. Smith, J. Iron Steel Inst., 20(11), 998 (1970)

9. Y.M. Dombrovskii, V.V. Mul'chin, V.N. Pustovoit, Steel Transl., 38, 105 (2008) 